\title{
Osteoarthritis prevention and meniscus regeneration induced by transplantation of mesenchymal stem cell sheet in a rat meniscal defect model
}

\author{
YIYING QI, GUANGNAN CHEN and GANG FENG \\ Department of Orthopedic Surgery, The Second Affiliated Hospital, School of Medicine, \\ Zhejiang University, Hangzhou, Zhejiang 310009, P.R. China \\ Received February 11, 2015; Accepted March 10, 2016
}

DOI: $10.3892 /$ etm.2016.3325

\begin{abstract}
Transplantation of mesenchymal stem cells (MSCs) is a potential therapy for meniscus regeneration. However, when using single cell suspension injection, there is frequently a significant loss of cells, with only a small percentage of cells remaining at the target site. This issue may be solved with the use of MSC sheets. In the present study, we investigated whether the use of MSC sheets were able to regenerate the meniscus effectively in a rat meniscectomized model. The anterior half of the medial meniscus in 10 rats was excised and an MSC sheet was transplanted in the MSC sheet treatment group, while untreated rats served as the control. After 4 and 8 weeks, the knee joints were examined by gross and histological observation. Histological observation revealed that the anterior portion of meniscus was similar to the native tissue, showing typical fibrochondrocytes surrounded by richer extracellular matrix in the MSC sheet group. In addition, predominant collagen-rich matrix bridging the interface was observed and the neo-meniscus integrated well with its host meniscus. Furthermore, degenerative changes of tibial plateau and femoral condyle occurred in the two groups. MSC sheet transplantation alleviated the degenerative changes efficiently. In conclusion, transplantation of MSC sheets may efficiently promote meniscus regeneration, as well as inhibit the progression of osteoarthritis in knee joints.
\end{abstract}

\section{Introduction}

The meniscus is a wedge-shaped fibrocartilaginous structure that is involved in shock-absorption, load distribution and knee joint stability. Due to its largely avascular nature, the meniscus

Correspondence to: Dr Gang Feng, Department of Orthopedic Surgery, The Second Affiliated Hospital, School of Medicine, Zhejiang University, 88 Jiefang Road, Hangzhou, Zhejiang 310009, P.R. China

E-mail: fngn@sina.com; qiyiying@aliyun.com

Key words: mesenchymal stem cell sheet, meniscus, osteoarthritis, transplantation, regeneration presents poor healing (1). The loss of meniscal function caused by meniscal tears, partial and total meniscectomy, and meniscal degeneration lead to the development or progression of knee osteoarthritis (OA) $(2,3)$. Previous studies have confirmed the direct association of partial or total meniscectomy with the development of OA. Therefore, it is necessary to repair the damaged meniscus with restoration of its structure and function.

For meniscal defects occurring subsequent to meniscectomy or meniscal degeneration, meniscal graft or artificial meniscus transplantation have been previously performed; however, these methods remain controversial due to the invasiveness, durability and safety of the transplant (4). In addition, the selection of the appropriate allograft size is challenging $(5,6)$. Therefore, a novel strategy is required for meniscus regeneration.

Mesenchymal stem cells (MSCs), which have high potential for proliferation and chondrogenesis, and display a multipotential differentiation capacity that allows development along the chondrogenic lineage, are a potentially attractive cell source for meniscus regeneration. Several studies have been performed using the intra-articular injection of MSCs and have shown promising results (7-9). Previous studies have reported that intra-articular injection of 5 million synovial MSCs promoted meniscal regeneration in rat models (10), whereas the injection of fewer cells did not result in meniscal regeneration (11). Furthermore, there is frequently a significant loss of cells when using single cell suspension injection, with only a small percentage of cells remaining at the target site. The use of MSC sheets may be a solution to these issues, since cell sheets not only provide a large number of MSCs, but can also be attached to host tissues and even wound sites, covering the surface with minimal cell loss via their deposited extracellular matrix (ECM).

The aim of the present study was to investigate the effect of MSC sheet transplantation for meniscus regeneration and OA prevention in a rat massive meniscectomized model.

\section{Materials and methods}

Isolation and culture of rat bone marrow-derived MSCs. All animal experiments were performed according to 'Guide for the Care and Use of Laboratory Animals' (National Institute of Health publication, 8th Edition 2011), as well as the standard 
guidelines approved by the Ethics Committee of Zhejiang University (Hangzhou, China).

Adult male Sprague-Dawley Rats $(\mathrm{n}=10)$ were obtained from the Experimental Animal Centre of Zhejiang University, (Hangzhou, China). The rats were bred and maintained under a 12-h-dark:light cycle with free access to food and a water. Room temperature was set at $25 \pm 3^{\circ} \mathrm{C}$ and relative humidity at $60 \pm 15 \%$. Rats $(n=2)$ were used as donors for transplantation of MSCs sheet, and 10 rats were used as recipients. Rats were euthanized with $\mathrm{CO}_{2}$ inhalation, and then the femurs and tibias were removed. The bones were washed in minimal essential medium $\alpha($ MEM- $\alpha)$ supplemented with $10 \%(\mathrm{v} / \mathrm{v})$ penicillin and streptomycin (Gibco; Thermo Fisher Scientific, Inc., Waltham, MA, USA). The ends of the femurs and tibias were cut away from the epiphysis, and the bone marrow was flushed out of the bone using $10 \mathrm{ml}$ medium in a syringe. Next, the cells were filtered through a $70 \mu \mathrm{m}$ cell strainer and centrifuged at $300 \mathrm{x}$ g for $5 \mathrm{~min}$. The cell pellet was resuspended in $10 \mathrm{ml}$ MEM- $\alpha$ supplemented with $10 \%$ fetal bovine serum (Thermo Fisher Scientific, Inc.) and plated in a culture plate. Cells were maintained at $37^{\circ} \mathrm{C}$ in a humidified atmosphere with $5 \% \mathrm{CO}_{2}$, and the medium was changed every 2 days. When adherent cells reached $80-90 \%$ confluence, they were detached with $0.25 \%$ trypsin-EDTA (Thermo Fisher Scientific, Inc.) and replated at a ratio of 1:3 in regular growth medium to allow for continued passaging. In order to ensure the consistency of experiments in vitro and in vivo, MSCs derived from rats bone marrow at passage 3 were used in all further experiments A maximum of 6 doublings of the cells were performed during the expansion and culturing procedures.

Cell sheet preparation. To create the cell sheet, the released cells were seeded at $4 \times 10^{4}$ cells $/ \mathrm{cm}^{2}$ into flasks cultured in $10 \mathrm{ml}$ of MEM- $\alpha$ supplemented with 10\% FBS (Gibco, Thermo Fisher Scientific Inc.) for a minimum of 1 week. Cells were maintained at $37^{\circ} \mathrm{C}$ in a humidified atmosphere with $5 \% \mathrm{CO}_{2}$, and the medium was replaced daily. After 1 week, the layered BMSCs were formed. The cells were then rinsed with phosphate-buffered saline (Gibco) twice, and then lifted as a cell sheet using a scraper (Fig. 1) (12). Cell proliferation was determined by DNA content assay using a fluorescent dye Hochest 33258 (Sangon Biotech Co., Ltd., Shanghai, China). The MSC sheets were harvested and stored at $-20^{\circ} \mathrm{C}$ until the assay was performed. For the DNA content assay, the cells were thawed at room temperature and homogenized in $1 \mathrm{ml}$ of lysis buffer $(50 \mathrm{mM}$ Tris- $\mathrm{HCl}, \mathrm{pH} 7.6$, $0.1 \% \mathrm{v} / \mathrm{v}$ Triton X-100; Sangon Biotech Co., Ltd.). The lysate was sonicated on ice for $30 \mathrm{sec}$. After centrifugation, the supernatant was collected for measurement. Cells $\left(\sim 4.8 \times 10^{6}\right)$ were contained within a single cell sheet, as determined by DNA quantification using an PerkinElmer LS 55 Fluorescence(PerkinElmer, Inc., Waltham, MA, USA) and the fluorescent dye, Hoechst 33258 (13).

Meniscectomy and MSC sheet transplantation. A total of 10 male Sprague Dawley rats (age, 12-14 weeks) were used, which were divided into the MSC sheet transplantation and untreated control groups. The knees of rats were randomly used for transplantation of MSC sheet or untreated. Rats were sacrificed at weeks $4(\mathrm{n}=5$ knees) and $8(\mathrm{n}=5$ knees) after surgery in the two groups. The rats were anaesthetized by injection of ketamine hydrochloride ( $2 \mathrm{mg} / \mathrm{kg}$ of body weight; China National

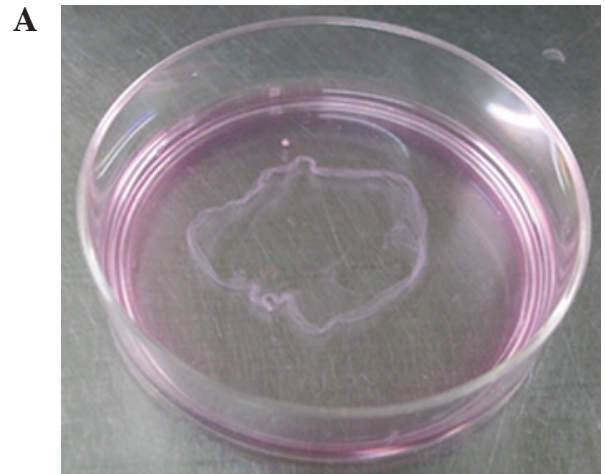

B

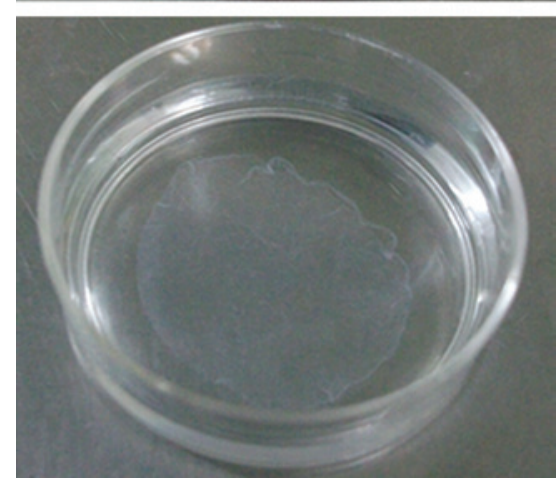

Figure 1. (A) Bone marrow-derived MSC sheet was harvested using a cell scraper. After the cell sheet formed, the cell sheet was able to be scraped from the edge and obtained by cell scraper. (B) The harvested MSC sheet spread in a cell culture plate. MSC, mesenchymal stem cell.

Medicines Corporation Ltd, Beijing, China) into the peritoneal cavity. Under anesthesia, a straight incision was performed on the anterior side of the bilateral knee, the anteromedial side of the joint capsule was cut, and the anterior horn of the medial meniscus was dislocated anteriorly with a forceps. The meniscus was then cut vertically at the level of medial collateral ligament, and the anterior half of medial meniscus was excised. Next, the dislocated meniscus was removed. The MSC sheet was transplanted into the knee joint of rats in the MSC sheet group, while no cells were transplanted in the untreated control group. Subsequently, after transplantation, fascia and skin were closed separately over the wound, and the rats were allowed to walk freely. At 4 and 8 weeks post-surgery, the rats were sacrificed by intraperitoneal injection of $3 \%$ chloral hydrate $(5 \mathrm{ml} / \mathrm{kg}$ body weight, Sangon Biotech Co., Ltd.).

Histological examination. Immediately after the rats were sacrificed, the femoral condyle, medial meniscus and tibial plateau from the two groups were collected and fixed in $4 \%$ paraformaldehyde for 3 days. The images of the tissues were obtained after 4 and 8 weeks. Next, tissues were decalcified in $20 \%$ EDTA solution for 14 days and then embedded in paraffin wax. The specimens were sectioned in a sagittal plane at $5 \mu \mathrm{m}$, and stained with safranin-o and fast green (both Sangon Biotech Co., Ltd.). Stained histological sections were observed using a BX53 microscope (Olympus Corp., Tokyo, Japan). The modified Pauli's histological scoring system was used to evaluate the regenerated meniscus, with the maximum score being 18 and lower scores indicating values closer to the normal meniscus (14). The degree and quality of cartilage were assessed and scored by an observer blinded to the study groups using 
A

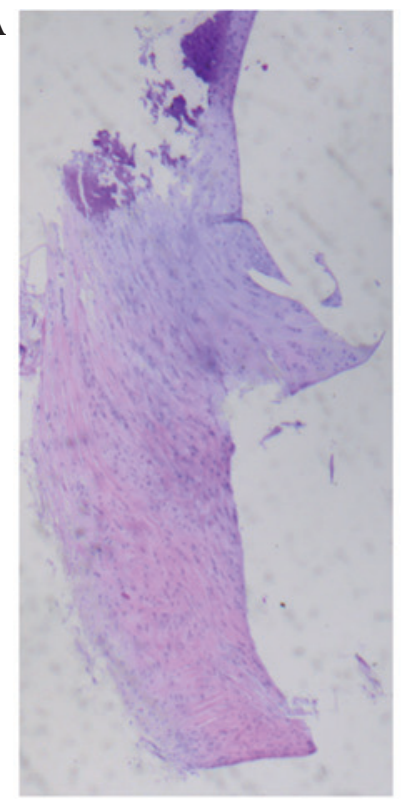

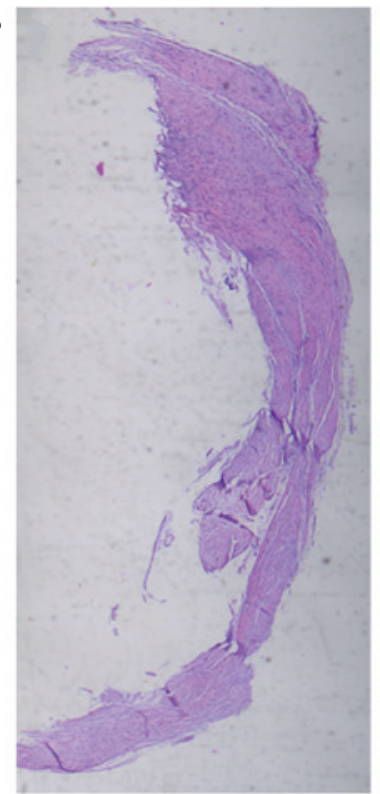
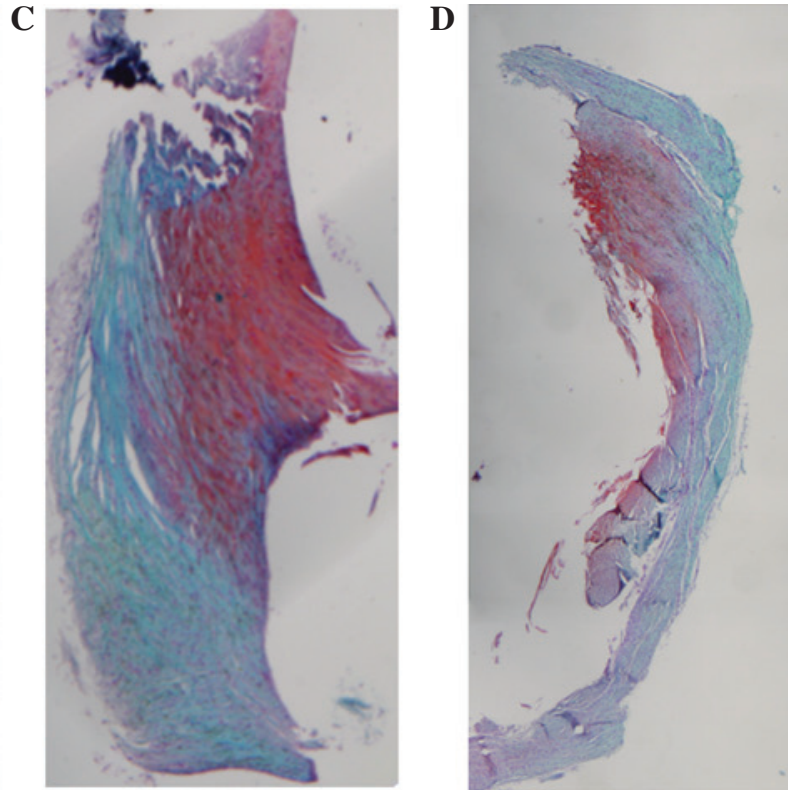

Figure 2. Histological observations of regenerated meniscal tissues at 4 weeks after surgery in the (A and C) control, and (B and D) MSC sheet groups. Staining in (A) and (B) was performed with hematoxylin and eosin, whilst (C) and (D) are stained with Safranine-O. Magnification, x20. MSC, mesenchymal stem cell.

A

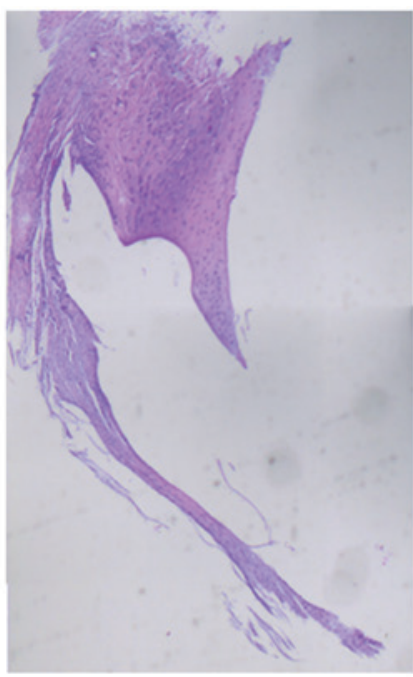

B

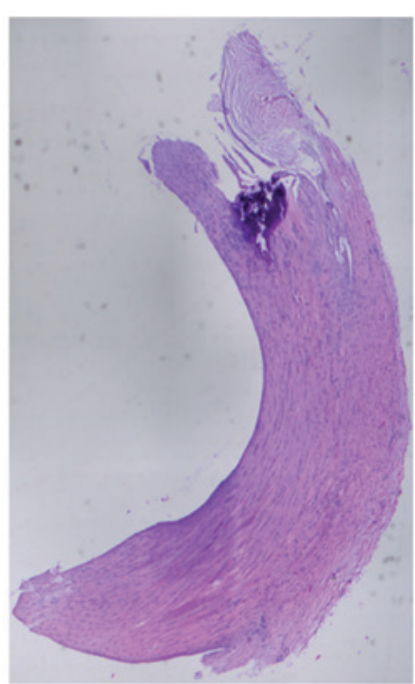

C

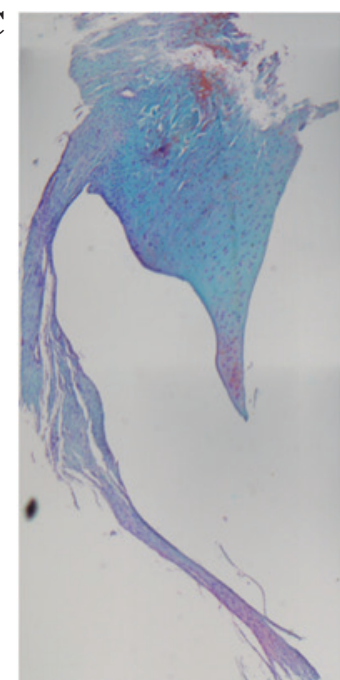

D

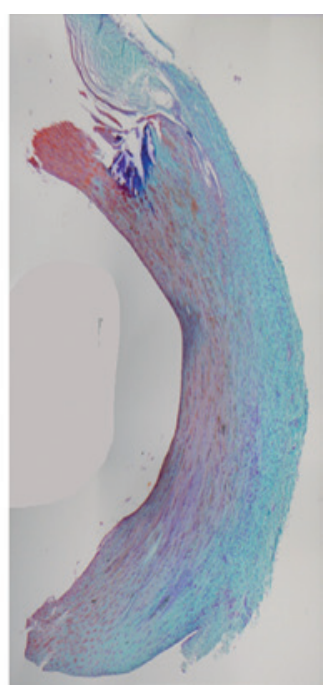

Figure 3. Histological observations of regenerated meniscal tissues at 8 weeks after surgery in the (A and C) control, and (B and D) MSC sheet groups. Staining in (A) and (B) was performed with hematoxylin and eosin, whilst (C) and (D) are stained with Safranine-O. Magnification, x20. MSC, mesenchymal stem cell.

a cartilage repair grading system, adapted from the study by O'Driscoll et al (15).

Statistical analysis. The obtained Pauli's scores were analyzed by Student's t-test and the statistically significant differences were determined at $\mathrm{P}<0.05$. Data analyses were performed using the SPSS software (version 15.0; SPSS, Inc., Chicago, IL, USA). Data are presented as mean \pm standard deviation.

\section{Results}

Clinical observations. No evidence of infection was observed in any of the animals prior to and following the experiments. All animals maintained their weight subsequent to surgery, and there was no evidence of local inflammation or immobilization of the joint.

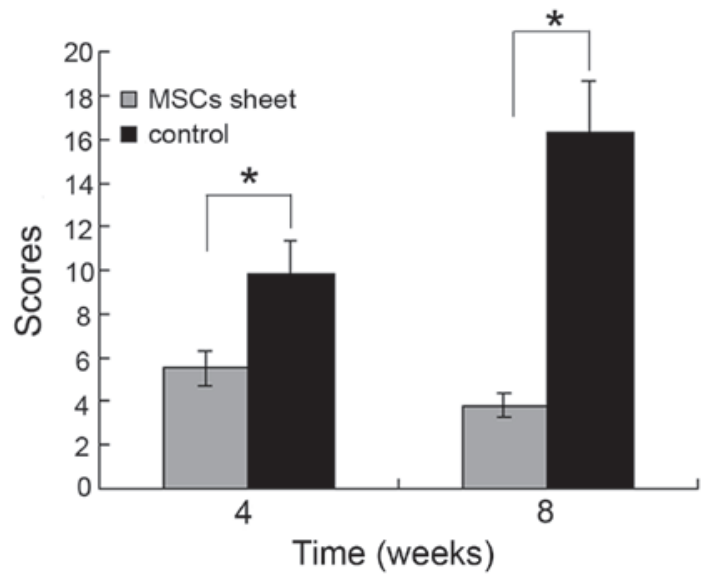

Figure 4. Histological scores for the regenerated meniscus in the MSC sheet and control groups ( $\mathrm{P}<0.05)$. MSC, mesenchymal stem cell. 

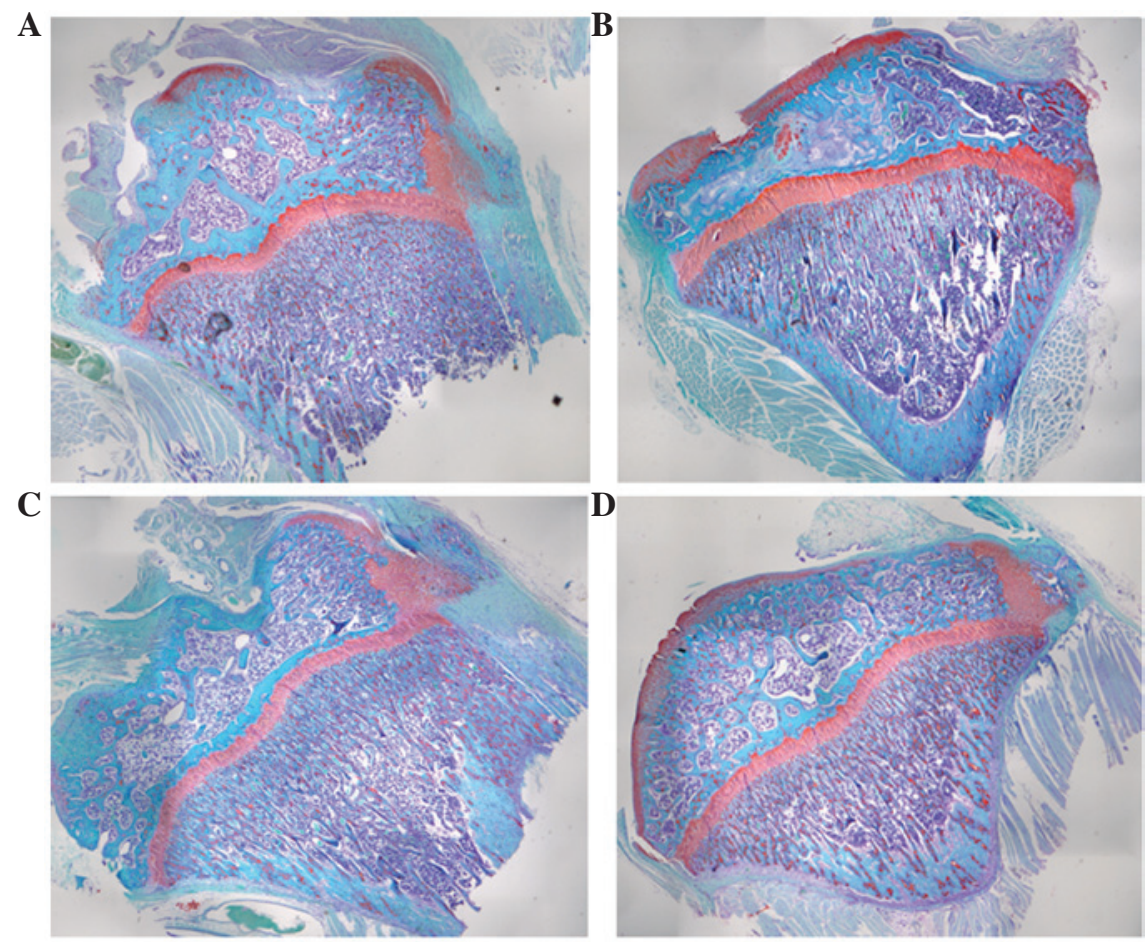

Figure 5. Safranine-O staining of the surface of tibial plateau in the (A) control and (B) MSC sheep groups at 4 weeks after surgery, and in the (C) control and (D) MSC sheep groups at 8 weeks after surgery. Magnification, x20. MSC, mesenchymal stem cell.
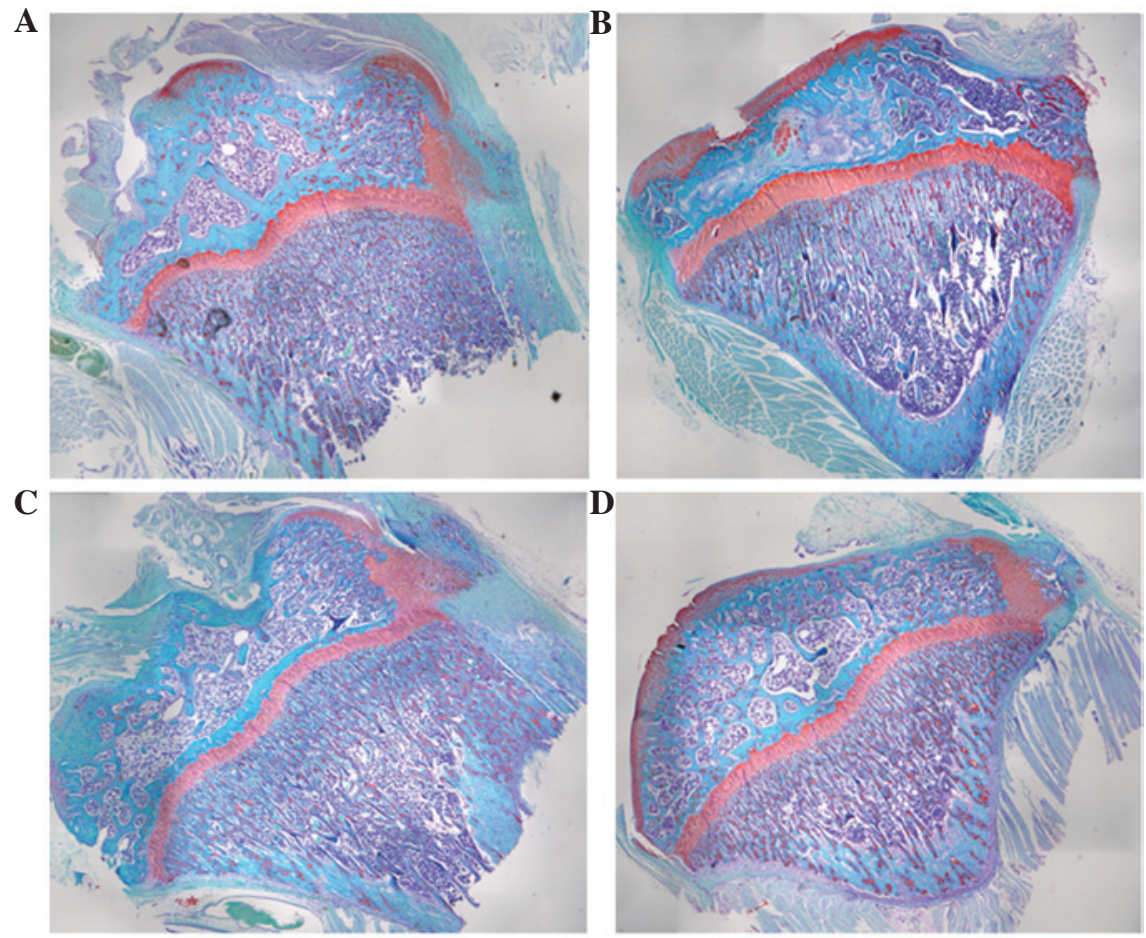

Figure 6. Safranine-O staining of cartilage from the femoral condyle region in the (A) control and (B) MSC sheep groups at 4 weeks after surgery, and in the (C) control and (D) MSC sheep groups at 8 weeks after surgery. Magnification, x20. MSC, mesenchymal stem cell.

Meniscal regeneration by transplantation of MSC sheet. At 4 weeks after surgery, the shape of the meniscus in the untreated group was not altered and even presented some atrophy at the border. In the MSC sheet group, the new hypercellular fibrocartilaginous tissue regenerated at the outside of the host meniscus, stained with rich glycosaminoglycan
(GAG) content in the matrix (Fig. 2). Positive area of S-O staining is indicative of GAG content

At 8 weeks after surgery, the meniscus appeared to be degenerated and atrophied in the control group. The meniscus size was smaller and filled with fibroblastic cells and a reduced amount of ECM. In addition, a thin meniscus-like 
tissue regenerated at outside of host meniscus. By contrast, in the MSC sheet group, the anterior portion of meniscus was regenerated, similar to the native meniscus and showed typical fibrochondrocytes surrounded by a richer ECM. Furthermore, predominant collagen-rich matrix bridging the interface was observed, and the neo-meniscus integrated well with its host meniscus (Fig. 3).

Histological scores. The histological scores for the regenerated meniscus in the MSC sheet group were found to be $5.5 \pm 0.8$ and $3.8 \pm 0.6$ at 4 and 8 weeks after surgery, respectively. These scores were significantly lower compared with those in the untreated control group, which were found to be $9.8 \pm 1.5$ and $16.3 \pm 2.4$ at 4 and 8 weeks after surgery, respectively $(\mathrm{P}<0.05$; Fig. 4$)$.

Prevention of cartilage degeneration by transplantation of MSC sheet. Beside meniscal regeneration, degenerative changes on the surface of the medial femoral condyle and medial tibial plateau were evaluated, such as cartilage erosion and osteophyte formation (Figs. 5 and 6). No chondral lesions were detected at the time of transplantation surgery. Subsequent to surgery, chondral damages on the surface of tibial plateau were detected, which were more severe compared with those on the surface of femoral condyle in the two groups.

At 4 weeks after surgery, a large area of focal articular lesions was seen on the surface of tibial plateau in the control group. Only a small area was covered by cartilage tissue (Fig. 5A). By contrast, in the MSC sheet-treated joints, no focal articular lesions were detected on the surface of tibial plateau at 4 weeks, and cartilage degeneration of the edge of tibial plateau was observed (Fig. 5B). At 8 weeks, the severity of tibial plateau chondral damage increased in the control group, with almost no cartilage observed on the surface of tibial plateau, while the surface was rugged (Fig. 5C). In the MSC sheet group, the surface of tibial plateau was slightly coarse, with some cracks and a decreased GAG content at 8 weeks after surgery (Fig. 5D).

In the femoral condyle region, the GAG content decreased gradually between weeks 4 and 8 in the two groups (Fig. 6). In the control group, the cartilage from the femoral condyle region exhibited apparent degeneration at 4 and 8 weeks (Fig. 6A and C). In the MSC sheet group, the cartilage from the femoral condyle region exhibited no apparent or slight degeneration at 4 weeks (Fig. 6B), while part of cartilage area appeared degenerated at 8 weeks.

Based on the ICRS scores (15) of the cartilage from the femoral condyle region, the MSC sheet-treated group presented higher scores $(18.5 \pm 3.3$ at 4 weeks and $14.9 \pm 2.4$ at 8 weeks) when compared with the control group (12.3 \pm 1.8 at 4 weeks and $9.6 \pm 0.6$ at 8 weeks). All the results indicated that transplantation of MSC sheet resulted in a certain degree of protection against OA development in the rats.

\section{Discussion}

In the present study, transplantation of MSC sheet was demonstrated to promote meniscus regeneration, while reducing efficiently $\mathrm{OA}$ of knee joint. Through the use of MSC sheet transplantation, the number of MSCs attached to the meniscal defect can be higher, compared with cell suspension. Furthermore, the MSC sheet is visible and can be placed around the meniscal defect directly, filling the space caused by meniscectomy and avoiding loss of MSCs from targeted defects.

Studies has previously reported the effect of injection of MSCs on the retardation of articular cartilage degeneration and meniscus regeneration in massive meniscectomized models of large animals $(16,17)$. However, cell suspension injection can cause cell loss and untargeted organ immigration. In the study of Mizuno et al (11), 1 million cells were intra-articular injected into the knee joint in a rat cylindrical meniscus defect model, and only a faint fluorescence signal was visible. Therefore, MSCs arranged in a three-dimensional structure of sheets or aggregates have an improved effect and were found to be superior to the use of cell suspensions. Katagiri et al (18) reported that transplantation of synovial MSC aggregates regenerated the meniscus more effectively compared with intra-articular injection of synovial MSCs using the same number of MSCs as a cell suspension in a rat massive meniscectomized model. The aforementioned results were in accordance with the findings of the present study, in which MSC sheet transplantation greatly promoted meniscus regeneration.

The MSC sheet is able to achieve highly efficient cell delivery (19) and effectively preserve the cell-cell interaction and the ECM (12). Through direct cell-cell interaction or the secretion of a broad spectrum of bioactive molecules with immunoregulatory and/or regenerative activities, the MSC sheet can exert a significant effect on local tissue repair by modulating the local environment and activating endogenous meniscus progenitor cells (20). Furthermore, the cells in the MSC sheet may be directly involved in meniscus regeneration due to the site-specific differentiation of MSCs (21).

When the meniscus is injured, the adjacent synovial tissue can be induced and contribute to the meniscal repair during the natural period of meniscal healing (22). This was also seen in the results of the present study. In the untreated control group, a thin meniscus-like tissue was regenerated on the outside of the host meniscus at 8 weeks, induced by synovial tissue. By contrast, in the MSC sheet group, a similar meniscus-like tissue formed at the outside of the host meniscus at 4 weeks after surgery. These findings suggest that the transplanted MSC sheet also expressed trophic factors and stimulated the adjacent synovial tissue, and subsequently progenitors of meniscus were induced, thus contributing to the meniscal regeneration.

In the current study, MSC sheet transplantation reduced the degree of cartilage degeneration, osteophyte formation and subchondral sclerosis, when compared with the control group, in accordance with the findings of previous studies of intra-articular injection of MSC suspensions $(17,23,24)$. Two possible reasons may explain the results. Firstly, the regenerated immature meniscus may protect the articular cartilage and suppress OA progression. In addition, the injected MSC sheet may produce trophic factors to inhibit progression of articular cartilage degeneration by inhibiting inflammatory cytokines.

In terms of clinical application, there are several limitations to the present study. Allogeneic MSC sheets were used for transplantation; however, autologous MSCs can be easily obtained from the bone marrow of patients, and then expanded in culture to construct cell sheets, which can be easily applied 
in clinical practice. Therefore, the use of MSC sheet is practically convenient for meniscal regeneration. However, the observation time in the current study was too short to evaluate the final outcome of the meniscus. The time period for neo-meniscus degeneration requires further investigation. Furthermore, tissue samples were only analyzed histologically in the present study, with no biomechanical tests performed on the regenerated meniscus, and thus the properties of the regenerated meniscus were not investigated. In spite of these limitations, the present study demonstrated a novel treatment of MSC sheet transplantation for regeneration of a massive meniscal defect. This method has the potential to regenerate the meniscus and inhibit the progression of OA.

In conclusion, transplantation of MSC sheets may efficiently promote meniscus regeneration, while also inhibit the progression of OA of the knee joint in a rat massive meniscectomized model. This transplantation technique using MSC sheets may have a great potential in meniscus regeneration in clinical practice.

\section{Acknowledgements}

The study was supported by grants from the Natural Science Youth Foundation of Zhejiang Province (no.LQ14H060001) and the Natural Science Youth Foundation of China (no. 81401779).

\section{References}

1. Englund M, Roemer FW, Hayashi D, Crema MD and Guermazi A Meniscus pathology, osteoarthritis and the treatment controversy. Nat Rev Rheumatol 8: 412-419, 2012.

2. Song Y, Greve JM, Carter DR and Giori NJ: Meniscectomy alters the dynamic deformational behavior and cumulative strain of tibial articular cartilage in knee joints subjected to cyclic loads. Osteoarthritis Cartilage 16: 1545-1554, 2008

3. Lohmander LS, Englund PM, Dahl LL and Roos EM: The long-term consequence of anterior cruciate ligament and meniscus injuries: Osteoarthritis. Am J Sports Med 35 1756-1769, 2007

4. Tucker B, Khan W, Al-Rashid M and Al-Khateeb H: Tissue engineering for the meniscus: A review of the literature. Open Orthop J 6: 348-351, 2012.

5. Lee AS, Kang RW, Kroin E, Verma NN and Cole BJ: Allograft meniscus transplantation. Sports Med Arthrosc 20: 106-114, 2012.

6. Lee DH, Kim JM, Lee BS, Kim KA and Bin SI: Greater axial trough obliquity increases the risk of graft extrusion in lateral meniscus allograft transplantation. Am J Sports Med 40: $1597-1605,2012$

7. Maumus M, Guérit D, Toupet K, Jorgensen C and Noël D: Mesenchymal stem cell-based therapies in regenerative medicine: Applications in rheumatology. Stem Cell Res Ther 2: 14, 2011.

8. Centeno CJ, Busse D, Kisiday J, Keohan C, Freeman M and Karli D: Increased knee cartilage volume in degenerative joint disease using percutaneously implanted, autologous mesenchymal stem cells. Pain Physician 11: 343-353, 2008.
9. Csaki C, Schneider PR and Shakibaei M: Mesenchymal stem cells as a potential pool for cartilage tissue engineering. Ann Anat 190: 395-412, 2008.

10. Koga H, Shimaya M, Muneta T, Nimura A, Morito T, Hayashi M, Suzuki S, Ju YJ, Mochizuki T and Sekiya I: Local adherent technique for transplanting mesenchymal stem cells as a potential treatment of cartilage defect. Arthritis Res Ther 10: R84, 2008.

11. Mizuno K, Muneta T, Morito T, Ichinose S, Koga H, Nimura A, Mochizuki T and Sekiya I: Exogenous synovial stem cells adhere to defect of meniscus and differentiate into cartilage cells. J Med Dent Sci 55: 101-111, 2008.

12. Nakamura A, Akahane $M$, Shigematsu $H$, Tadokoro $M$, Morita Y, Ohgushi H, Dohi Y, Imamura T and Tanaka Y: Cell sheet transplantation of cultured mesenchymal stem cells enhances bone formation in a rat nonunion model. Bone 46: 418-424, 2010.

13. Hong Y, Song H, Gong Y, Mao Z, Gao C and Shen J: Covalently crosslinked chitosan hydrogel: Properties of in vitro degradation and chondrocyte encapsulation. Acta Biomater 3: 23-31, 2007.

14. Pauli C, Grogan SP, Patil S, Otsuki S, Hasegawa A, Koziol J, Lotz MK and D'Lima DD: Macroscopic and histopathologic analysis of human knee menisci in aging and osteoarthritis. Osteoarthritis Cartilage 19: 1132-1141, 2011.

15. O'Driscoll SW, Keeley FW and Salter RB: The chondrogenic potential of free autogenous periosteal grafts for biological resurfacing of major full-thickness defects in joint surfaces under the influence of continuous passive motion. An experimental investigation in the rabbit. J Bone Joint Surg Am 68: 1017-1035, 1986

16. Murphy JM, Fink DJ, Hunziker EB and Barry FP: Stem cell therapy in a caprine model of osteoarthritis. Arthritis Rheum 48: 3464-3474, 2003.

17. Al Faqeh H, Nor Hamdan BM, Chen HC, Aminuddin BS and Ruszymah BH: The potential of intra-articular injection of chondrogenic-induced bone marrow stem cells to retard the progression of osteoarthritis in a sheep model. Exp Gerontol 47: 458-464, 2012.

18. Katagiri H, Muneta T, Tsuji K, Horie M, Koga H, Ozeki N, Kobayashi E and Sekiya I: Transplantation of aggregates of synovial mesenchymal stem cells regenerates meniscus more effectively in a rat massive meniscal defect. Biochem Biophys Res Commun 435: 603-609, 2013.

19. Yang J, Yamato M, Kohno C, Nishimoto A, Sekine H, Fukai F and Okano T: Cell sheet engineering: Recreating tissues without biodegradable scaffolds. Biomaterials 26: 6415-6422, 2005.

20. Koelling S and Miosge N: Stem cell therapy for cartilage regeneration in osteoarthritis. Expert Opin Biol Ther 9: 1399-1405, 2009.

21. Chen FH and Tuan RS: Mesenchymal stem cells in arthritic diseases. Arthritis Res Ther 10: 223, 2008.

22. Katagiri H, Muneta T, Tsuji K, Horie M, Koga H, Ozeki N, Kobayashi E and Sekiya I: Transplantation of aggregates of synovial mesenchymal stem cells regenerates meniscus more effectively in a rat massive meniscal defect. Biochem Biophys Res Commun 435: 603-609, 2013.

23. González MA, Gonzalez-Rey E, Rico L, Büscher D and Delgado M: Treatment of experimental arthritis by inducing immune tolerance with human adipose-derived mesenchymal stem cells. Arthritis Rheum 60: 1006-1019, 2009.

24. Tuan RS: Stemming cartilage degeneration: Adult mesenchymal stem cells as a cell source for articular cartilage tissue engineering. Arthritis Rheum 54: 3075-3078, 2006. 